\title{
¿De qué se habla cuando se habla de territorio?
}

Analía Soledad D'Angelo

Licenciada. en Sociología (Universidad

Nacional de Buenos Aires)

Magister en Hábitat y pobreza urbana en

América Latina (Universidad Nacional de

Buenos Aires)

Doctora. en Ciencias Sociales (Universidad

Nacional de Buenos Aires)

Correo electrónico: analiadangelo@gmail.com 


\section{Resumen}

El presente artículo resulta una primera aproximación al estado del arte existente dentro de la producción teórica de las Ciencias Sociales durante las últimas décadas, en torno a la noción de "territorio" y, al mismo tiempo, un ejercicio teórico de explicitación de la vinculación de dicha noción con las líneas de investigación en curso del equipo en el que la autora se inscribe. Lo motiva la necesidad de revisión y especificación de un concepto nodal para muchas investigaciones del ámbito de nuestras disciplinas en las que el mismo es utilizado y tratado de modo vago, polisémico y sin mayores precisiones teórico conceptuales que permitan posicionarse o distanciarse de acepciones que no se adecúen a la propia perspectiva. Se intenta aportar a la comprensión del postulado sostenido por el PEC que expresa que la "economía popular" -universo de estudio sobre el que versan nuestras líneas de investigación- se encuentra territorializada. Asimismo, procuraremos despuntar algunos aportes que desde las ciencias sociales pueden ofrecerse específicamente tanto a los profesionales dedicados a la sociología urbana como a la arquitectura y el urbanismo, en lo referido a pensar el territorio y las consecuencias prácticas que toda perspectiva teórica por la que se opta conlleva.

\section{Palabras clave}

territorio, territorialidad, ciencias sociales, urbanismo

\section{Summary}

The present article is a first approximation to the existing academic production of the social sciences during the last decades, around the notion of "territory" and, at the same time, a theoretical exercise of explanation of the link between this notion and the research lines of the academic group in which the author enrolls. It is motivated by the need of revision and specification of a nodal concept for many researches in the field of our disciplines in which it is used and treated in a vague, polysemic way, and without major theoretical precision that allows positioning or distancing from meanings that don't reflect researches's perspective. We try to contribute to the understanding of the postulate sustained by the PEC that expresses "popular economy" -the universe on which our research lines are basedis territorialized. Also, we will try to highlight some contributions from the social sciences that can be offer specifically to professionals dedicated to urban sociology as well as to architecture and urbanism, in what refers to thinking territory and practical consequences of the perspective we adopt.

\section{Keywords} territory, territoriality, social sciences, urbanism 


\section{¿Qué es el territorio?}

Disponerse a abordar las nociones de territorio y de territorialidad nos conduce a un tema de estudio sobre el cual no ha habido ni hay consenso. Originalmente, es posible rastrear que ambos términos comenzaron a ser utilizados en el ámbito de la geografía.

Alain Faure (2009:418, citado en Jolly, 2012:2), en la sección dedicada al concepto de territorio/territorialización en su Diccionario de políticas públicas, comienza del siguiente modo: "Desde hace algunos años, en las ciencias sociales, se evidencia que las referencias al territorio o a sus derivados tienen un uso bastante problemático debido al sentido incierto que éstas revelan. El término genera a menudo acepciones ambiguas en los discursos expertos: las invocaciones recurrentes y reversibles a los "proyectos de territorio" y a los "territorios de proyecto", son ilustraciones de lo anterior, así como el calificativo comodín "territorial", sobre-utilizado para atribuirle todas las virtudes de los términos de gobernanza, legitimidad, equidad, desarrollo, continuidad, liderazgo o excelencia. (...) La noción de 'territorio' posee un estatus ambiguo. Es a la vez omnipresente en los debates acerca de los asuntos de poder o de las instituciones y casi nunca teorizado en sí mismo en las ciencias de lo político en Francia". Se trata de una inquietud similar a la que nos condujo a iniciar esta revisión.

Por su parte, el geógrafo Jérome Monnet expresa una mirada que se corresponde con la compartida por Faure. Para Monnet “... el primer desafío de una reflexión sobre el territorio es que aquel corresponde a una noción común utilizada sin ninguna necesidad de definirla por numerosísimas personas en una gran variedad de situaciones, mientras que su estudio se inscribe en unas conceptualizaciones finalmente muy diferentes (...). La variedad de usos de esta [noción] en los discursos político-administrativos, mediáticos, artísticos o científicos restringen la pretensión de ser exhaustivo o incluso de sintetizarlos” (Monnet, 2010:91-92, citado en Jolly, 2012:2).

Para comenzar a aproximarse al tema, tanto Faure como Monnet se dirigen en primer lugar hacia la etimología de la palabra. De este 
acercamiento, surgen dos raíces posibles: la primera relaciona territorio con "terror" y la segunda lo relaciona con "tierra”. Para Faure “...no es casualidad que la raíz latina de la palabra "territorio", jus terrendi, se refiera a aquel que detiene el derecho de aterrorizar. La noción de territorio está históricamente ligada a relaciones de poder y dominación. El término, extraño hasta el siglo XVII, va a permitir calificar el paso de los límites a las fronteras, es decir, de un espacio dado a un territorio dominado" (Faure, 2009:419, citado en Jolly, 2012:2).

En otros términos, Bertrand Badie citando a R. D. Sack (2001:300, citado en Jolly, 2012:3), indica que “...el territorio se analiza en la ciencia política como un constructo social, es decir, como el resultado de un intento hecho por un individuo o un grupo de afectar, influenciar o regir a unas personas, fenómenos o relaciones, delimitando y controlando un área geográfica”.

Por su parte, los geógrafos colombianos Montañez y Delgado (1998:122-123, citados en Jolly, 2012:3) indican que el territorio es reconocido como un espacio de poder y de dominio de distintos sectores, que incluyen al Estado, a los individuos, a distintos grupos, organizaciones y a empresas multinacionales.

Para Monnet (2010:92-93, citado en Jolly, 2012:3) la etimología de la palabra territorio remite al latín territorium, en la cual “...la raíz 'terra' remite a la tierra y el sufijo 'torium' significa “el lugar en el cual se desarrolla la acción o el instrumento que sirve a cumplir la acción”. El territorio es entonces etimológicamente el encuentro de la materia y de la acción, del objeto sobre el cual se actúa y del sujeto que actúa. En esta perspectiva, Monnet lo define entonces como "el espacio material, área o red, realizado por el ejercicio de una acción humana repetitiva" (Jolly, 2012:3).

A su vez, muchos estudios y producciones de las ciencias sociales de los últimos años, toman la cuestión del territorio con el objetivo de analizar -tanto desde un punto vista teórico como operativo- el rol jugado por el mismo en procesos de desarrollo local del pasado y la actualidad, o si se quiere, a los fines de interpretar el desarrollo local desde una perspectiva territorialista (Dematteis y Governa, 2005). 
Desde estas miradas que se centran en pensar el rol del territorio en el desarrollo local, Dematteis y Governa afirman: “...es sustancialmente compartido que la creciente internacionalización de la economía, la caída de las barreras que antes limitaban la extensión geográfica de los contornos, la intensificación de las interacciones de larga distancia y de la interdependencia entre los lugares, la invasión de la competencia y de las ideologías conexas, y el cambio de la relación entre los factores móviles e inmóviles del desarrollo, ha dado origen a un modo diverso de considerar el territorio, con una creciente atención al nivel local. Más controvertido resulta comprender cuál es el papel del territorio en el interior de tales procesos" (Amin, 2002, citado en Dematteis y Governa, 2005:32).

Las preguntas por el lugar del territorio en el desarrollo local implican muchas otras, a modo ilustrativo: ¿cuáles son las relaciones entre las dinámicas locales y las dinámicas globales?, ¿cómo aplicar el desarrollo local?, ¿cuáles son los instrumentos más adecuados para conseguirlo?, ¿cuál es la relación entre las diversas dimensiones del desarrollo? (económica, social, cultural, política, etc.), etc. Sin embargo, consideramos que, en esa línea de indagación, la pregunta que se impone en primer lugar, previa a las anteriores, es la que se interroga acerca del "desarrollo local” en sí mismo, es decir, la que intenta definirlo. Otras líneas de investigación que también se interrogan por la relación entre territorio y desarrollo local, entienden al lugar/ territorio como conjunto de relaciones sociales territorializadas. Este último aspecto será retomado más adelante en nuestro desarrollo.

Finalmente, en este mismo escrito, los autores expresan: "la atención a la interacción entre sociedad/ambiente, territorio/territorialidad, a la centralidad de lo local, al concepto de lugar y a la importancia recobrada de la organización regional, hacen referencia a algunos de los temas propios de la reflexión geográfica moderna (...). Merece particular atención en esta perspectiva la redefinición de la relación sociedad/ambiente y, en particular, la reflexión sobre la dimensión comunitaria de las relaciones entre los actores y los actores con su territorio" (Dematteis y Governa, 2005:36). 
Muy próximo al concepto de territorio, nos encontramos con el concepto de territorialidad. Junto con Dematteis y Governa (2005:43), podemos decir que la territorialidad ha sido estudiada en dos campos de investigación.

El primero de ellos, lo constituye el estudio de la territorialidad como atributo básico del estado moderno, junto a la soberanía, como otro atributo fundamental. "La legitimidad y la acción política del estado moderno están así, basados en la soberanía nacional y en la imposición de una forma específica de la territorialidad sobre el territorio estatal (Allies, 1980 citado en Dematteis y Governa, 2005:43). El segundo campo de investigación estudia la territorialidad en un sentido originariamente biológico, es decir, en la transposición al campo humano de los estudios de la etología sobre los comportamientos territoriales de los animales. "Es como consecuencia de la confrontación con las posiciones que subrayan la naturaleza al mismo tiempo objetiva y subjetiva de los lugares (Entrikin, 1991 citado en Dematteis y Governa, 2005) que el debate sobre la territorialidad supera progresivamente una idea de territorialidad basada en la analogía entre territorialidad animal y territorialidad humana. De este modo se asienta progresivamente una concepción de territorialidad como componente geográfico clave para comprender cómo la sociedad y el espacio están íntimamente unidos. Es esta concepción relacional de territorialización, según la cual esta constituye la expresión geográfica del ejercicio del poder, un poder que se expresa territorialmente" (Dematteis y Governa, 2005:43).

Dentro de dicho marco general, las teorizaciones principales a la cuestión de la territorialidad pueden ser reagrupadas en torno a las posiciones de Sack y Raffestin. Para Sack, la territorialidad puede ser definida como "la tentativa de un individuo o de un grupo de influir o controlar las personas, los fenómenos y las relaciones, delimitando y ejerciendo un control sobre un área geográfica. Esta área se llamará territorio” (Dematteis y Governa, 2005:43). Para Raffestin, la territorialidad es un conjunto de relaciones que nacen en un sistema tridimensional "sociedad, espacio, tiempo", con el propósito de alcanzar la mayor autonomía posible compatible con los recursos del sistema (...) La territorialidad es 
el conjunto de las relaciones que una sociedad, y por tanto los individuos que forman parte de ella, mantienen con la exterioridad (las relaciones verticales con el territorio, el milieu, el ambiente) y con la alteridad (las relaciones horizontales con los demás agentes) para satisfacer las propias necesidades con la ayuda de mediadores" (Dematteis y Governa, 2005:44). Coincidimos con los autores en que, en este último caso, la territorialidad no es el resultado del comportamiento humano sobre el territorio, sino que es el proceso de construcción de tal comportamiento, el conjunto de las prácticas y de los conocimientos de los hombres en relación a la realidad material, las relaciones mantenidas de un agente con el territorio y con los demás agentes.

Ambas concepciones de territorialidad implican dos distintas y un tanto opuestas concepciones teóricas y disímiles orientaciones metodológicas.

Por su parte, para Monnet (2010:92 citado en Jolly, 2012:3), la territorialidad a partir del siglo XIX es "la capacidad de un actor de ejercer una competencia sobre alguna extensión”. Vale decir que su concepto se encuentra próximo al del politólogo Sack. En el siglo XX, agrega Monnet, los especialistas de etología animal retoman el concepto, pero cambiándole el sentido que para ellos designa, entendiendo por ello "las relaciones que una especie entretiene con un territorio de subsistencia o de reproducción”. Hoy en día, concluye Monnet, "la evolución del sentido de territorialidad conduce a entenderla como un valor o un sistema de valores que unos actores sociales le asignan a un territorio determinado, y aún como el 'sentimiento de pertenencia' a aquel” (Jolly, 2012:3).

Hasta aquí hemos sintetizado algunas conceptualizaciones de territorio y territorialidad que provienen de perspectivas que pertenecen al ámbito de la geografía y de la ciencia política.

\section{Aproximaciones críticas a la noción de territorio}

Desde el campo de la sociología, el sociólogo Flabián Nievas, destaca cómo el concepto de espacio, y su derivado, territorio, tienen centralidad en las principales corrientes sociológicas clásicas. Postula 
que tanto la sociología comprensivista (cuyo padre fundador es Max Weber) y el marxismo (basado en las ideas de Marx) dan a estas nociones una importancia heurística fundamental si bien no encontraremos en sus obras una teoría espacial formulada. Dice Nievas, "en el caso del marxismo, no se podría pensar la circulación (transporte) ni la renta o la cooperación sin el espacio". En el caso de la sociología comprensivista, "un elemento de importancia decisiva como el Estado queda sellado al concepto de territorio". Coincidimos con el autor en que muchos estudios sociales han abordado el problema del espacio, especialmente, desde el campo de la sociología urbana. Sin embargo, no podemos afirmar lo mismo en lo que refiere a la noción de territorio, muy utilizada por el mencionado campo más no del mismo modo, explicitada. En tanto "territorio es espacio social", expresa Nievas (1994:2), esto ha hecho que ambos conceptos se utilicen indistintamente.

En su artículo, Nievas retoma al sociólogo Juan Carlos Marín y nos alerta sobre la concepción que tenemos de espacio, una noción que expresa "que ha estado dominada" por el elemento central, hegemónico de la concepción del mundo de las clases dominantes" (Marín, 1984:53, citado en Nievas, 1994:2). "De modo que se han constituido imágenes y formas de conocimiento propias de las clases dominantes y que son patrimonio del conjunto de las clases dominadas". Nievas propone tres series para abordar teóricamente la cuestión del territorio. Las mismas son: social, filogenética y ontogenética.

Sintetizaremos lo más fielmente posible cada una de estas series. La primera de ellas alude a lo que Nievas llama "el territorio burgués". Como bien expresa el autor, el capitalismo, respecto de otras formaciones sociales previas, reorganiza el tiempo y el espacio, y en relación a este último, lo hace en dos niveles: distinguiendo ámbitos de producción de mercancías (industria) y ámbitos de concurrencia para el intercambio de mercancías (mercado). Si bien la industria y el mercado son preexistentes al capitalismo, la articulación lograda por éste es novedosa. "El territorio es, entonces, la organización social y conceptual de un espacio, o para expresarlo, en otros términos, la construcción social de un espacio, la articulación de relaciones so- 
ciales con su asiento material y su inteligibilidad" (Nievas, 1994). A su vez, "el territorio se caracteriza por su homogeneización interna (que no significa identidad sino articulación en torno a determinada legalidad social) y su especificidad externa, su heterogeneidad respecto de otros territorios". La producción en el capitalismo se concentra económica y espacialmente. A fines del siglo XVIII e inicios del siglo XIX se constituyen los estados nacionales formalmente definidos. "De este modo se articula la relación de dominación, relación entre desiguales, y la relación de intercambio entre iguales. La primera al interior de cada Estado, la segunda, interestatal. Nace la patria y el ciudadano libre. De modo que esta territorialidad es esencialmente burguesa: El estado, la nación, son ahora el territorio de dominación de una burguesía dada. En el caso de la Argentina, "los territorios de las burguesías menos fortalecidas serán las provincias” (Nievas, 1994:4).

La segunda de las series que analiza Nievas tiene que ver con la construcción de un saber, un saber sobre el territorio. El autor va dando cuenta de los orígenes de la cartografía, motivada por intereses de comercio y de dominio. "Si bien los elementos de la geometría euclideana posibilitaban la triangulación, el impulso general que dio la geometría analítica cartesiana influyó (probablemente de modo decisivo) en el nacimiento de la cartografía "científica". El entrecomillado responde a la necesidad de poner de relieve el carácter estratégico de los corpus de conocimiento construidos, siempre desde una perspectiva de poder. Luego, a mediados del siglo XVIII comenzó el cartografiado de Francia por triangulación. Posteriormente, el nacimiento de la escuela geográfica alemana le dio un gran impulso al desarrollo de la cartografía científica y sentó los cimientos de la geopolítica o estudio de las relaciones de fuerza entre territorios.

A su vez, Nievas señala el surgimiento de la escuela francesa de geografía, la cual funda el concepto-obstáculo (así denominado por el autor) de región "engarzando distintos elementos cuyo efecto es el diseño de un paisaje geofísico, pero esta operatoria muy sutilmente ha dejado de lado la historia; nos presenta como una determinación natural a las relaciones sociales que lo conformaron" (1994, 6-7). 
Finalmente, la última serie desde la cual Nievas se sumerge en la problematización del concepto de territorio, es la ontogénesis. En este apartado, entre otros, afirma que "el fuerte anclaje que tiene el territorio en las personas está dado porque ese territorio no es el terreno, sino las relaciones sociales que allí se asientan y lo articulan, lo integran como paisaje en la necesaria relación hombre-naturaleza, de la que no puede prescindir. Y las relaciones sociales son, se sabe, la humanización misma del hombre" (Nievas, 1994:8).

Luego de este desarrollo, Nievas llega a desarrollar una definición de territorio, con la cual: "El territorio, pues, no es la superficie terrestre con todas sus rugosidades y especificidades, incluidos sus elementos minerales, suelos, vida vegetal y animal, clima, topografía, etc. (Coraggio, 1989, citado en Nievas, 1994:9), tal como lo describe la mirada corriente, largamente construida, sino la articulación de dos órdenes diferentes: de un lado, lo que a los efectos de diferenciarlo del concepto de espacio, de mayor abstracción, denominaremos como geoterritorio o porción de suelo: sumatoria de las condiciones biofísicas de una porción de la corteza del planeta, siendo la totalidad de ésta, el suelo; de otro, la totalidad de las relaciones sociales establecidas en y ordenadoras de dicho geoterritorio, fuera del cual no tienen existencia y al cual incorporan, no como continente sino como componente" (Nievas, 1994:9). La incorporación de la noción de geoterritorio establece la distinción entre las condiciones sociales y las naturales. Por su parte, el geoterritorio tiene un vínculo e influencias activas sobre el territorio, aunque no lo determina.

Afirmar que el territorio es social no implica negar el elemento/s natural, pero éste es insuficiente para determinarlo. "Lo social incorpora lo natural articulándolo en otro nivel: lo puramente natural con lo puramente humano" (Nievas, 1994:10).

Por último, nos resulta interesante rescatar otra cita del autor: "Se puede afirmar que hay territorios capitalistas, o que el capitalismo en su conjunto expresa un territorio; pero no que el capitalismo es igual a territorio (...) El feudalismo es demostrativo de la territorialidad fraccionada: cada feudo expresaba un territorio más o menos dife- 
renciado del resto. El capitalismo, en cambio, unifica espacios en un territorio común: el mercado" (Nievas, 1994:12).

Luego, el autor señala que el tiempo es una cuarta dimensión que hay que incorporar a la noción de territorio. Al momento, el territorio está compuesto por cuatro elementos: geoterritorio, formas de vinculación, sujeto y tiempo.

Finalmente, el autor establece una gradación de las cuatro dimensiones que presenta como constitutivas del territorio, ya que las mismas no tendrían el mismo nivel epistemológico. La más importante de ellas es la forma de vinculación existente en un territorio ("constituye la argamasa que dará cuerpo e identidad a la fuerza social (sujeto, personificado en cuerpos)" (Nievas, 1994:16).

Por su parte, Claudia Tomadoni (2008) ha escrito un interesante artículo en torno a las nociones de espacio-tiempo, espacio y territorio, agentes sociales y sustentabilidad. En el mismo, expresa: "El hecho de recurrir al tiempo solo como un factor explicativo y no como parte inherente del espacio es fuente de un equívoco singular: considerar al espacio como un escenario en el cual transcurren los hechos. Decir espacio significa decir proceso, lo cual implica decir tiempo. Por lo tanto, el espacio es un proceso social construido a través del espacio-tiempo" (Tomadoni, 2008:54). "En efecto, el espacio es proceso, es cambio y por tanto no puede entenderse de manera estática. Es una instancia dinámica, es pura acción y mutación de su naturaleza original, en nuevas formas espaciales o nuevas naturalezas que se construyen a través del tiempo (Santos, 1996a; 1996b, citado en Tomadoni, 2008:55)”. Este dinamismo, según la autora, solo puede interpretarse bajo la conjugación balanceada de tres dimensiones de la vida humana: espacialidad, historicidad y socialidad (Soja, 1994, citado en Tomadoni, 2008). Esta articulación da lugar a procesos en los cuales las preguntas acerca del "cuándo" y "quiénes" se responden necesariamente considerando el "dónde".

Aquí resulta relevante entender al espacio como una construcción, una producción, un constante hacer sobre formas ya construidas que nos habla de "un hacer" que va cambiando. El concepto de formación socioespacial incluye al espacio en la medida en que coadyuva a 
la reproducción de las relaciones sociales (relaciones no solo de producción sino de identidad, género, edad, procedencia, preferencias, intereses y valores de los agentes). Podemos decir junto con Tomadoni que "la reproducción en estos términos genera relaciones sociales, que tienen un correlato territorial específico que en determinadas coordenadas de tiempo y lugar da lugar a la conformación de un territorio" (Tomadoni, 2008:56). En sí, lo que la autora dice es que "el territorio no es un objeto, ni es una cosa, por el contrario, es un conjunto de relaciones complejas". Por otra parte, ofrece un aporte para pensar la relación espacio-tiempo: "podría aseverarse -expresa- que el espacio es tiempo condensado". Es así como sería posible reconstruir en los espacios y territorios las "marcas del pasado" y nutrirnos de la información que ello nos brinda para una comprensión más cabal de los procesos pasados y presentes de un determinado territorio.

Asimismo, esta autora distingue del siguiente modo el concepto de espacio del de territorio: "hablar de espacio es referirse a ámbitos construidos directa o indirectamente por la sociedad. Esta noción alude a un ámbito genérico que se concreta en un territorio. Así, el territorio es un lugar preciso, con límites y con características específicas según posibilidades e intereses de los diferentes agentes sociales dispuestos al juego de la construcción de ese territorio. En definitiva, el territorio es un constructo social en determinadas coordenadas de tiempo y lugar" (Tomadoni, 2008:58). Es una porción asequible del espacio geográfico.

Otro autor que cita Tomadoni, llamado Santos, postula en algunos trabajos que el territorio se define en la relación que se establece entre objetos geográficos (sociales y naturales) (...) y los procesos sociales (económicos, sociales, institucionales, religiosos, políticos, ideológicos, científico tecnológicos) que constituyen sistemas de acciones y flujos que como haz de fuerzas sociales se ejercen en un lugar y conforman la localización" (Santos, 1986 y 2000, citado en Tomadoni 2008:58).

Finalmente, otro de los aportes del texto de Tomadoni es aquel que aborda la cuestión de lo ofrecido -en términos teóricos y de análisis- por la teoría de la praxis al análisis geográfico. Como una sín- 
tesis más que ajustada de ello podemos decir junto con la autora que "los agentes sociales producen en su devenir el territorio" (Tomadoni, 2008:59).

\section{Algunas reflexiones que emergen desde la teoría a la práctica}

Vale decir que la cuestión principal de interés y que se aborda en las distintas líneas de investigación del PEC es la economía popular, sus características y dinámicas. A modo de ofrecer, en el marco de este artículo, una definición no exhaustiva y que obvie los debates contemporáneos acerca del sentido del término, diremos que la economía popular es la forma que adopta la economía en los territorios habitados por los sectores populares bajo estudio.

Otro de los conceptos fundamentales involucrados en nuestras investigaciones es el de estrategias de reproducción. Para el desarrollo del mismo nos basamos en la conceptualización que realiza la investigadora Alicia Gutiérrez, quien a su vez abreva en el trabajo intelectual de Pierre Bourdieu. La autora define a las estrategias de reproducción como un conjunto de prácticas fenomenalmente muy diferentes, por medio de las cuales los individuos y las familias tienden, de manera consciente o inconsciente, a conservar o aumentar su patrimonio, y correlativamente a mantener o mejorar su posición en la estructura de las relaciones de clase (Gutiérrez, 2003). Vale decir que "patrimonio" refiere al conjunto de bienes económicos, sociales, culturales y simbólicos, que los agentes o grupos de agentes poseen. Es decir que, el estudio de las estrategias de reproducción no se reduce al análisis de las prácticas que llevan a una repetición de las condiciones objetivas de vida, sino que incluye la observación del proceso de creación de condiciones nuevas y modificación duradera de las condiciones de existencia. Como expresa la autora, implica rescatar la capacidad activa e inventiva de la práctica y las capacidades generadoras del hábitus. Las condiciones estructurales, desde la perspectiva de Bourdieu (que adoptamos), no eliminan todo margen de autonomía y creatividad de los agentes sociales y con ello, la posibilidad de modificarlas. 
Uno de los supuestos fuertes de nuestro equipo -hipótesis que los sucesivos trabajos de campo van corroborando- tiene que ver precisamente con el hecho de observar que la matriz de estrategias que componen la economía popular, no puede explicarse por fuera de su inscripción territorial y en nuestro análisis involucra el concepto de matrices político-territoriales. Este último resulta muy importante en los desarrollos teóricos hasta aquí realizados por el PEC y representa un modo particular de conceptualizar al territorio.

Podremos decir entonces que el concepto de territorio supone, en primer lugar, la articulación entre estructura espacial y estructura social. Puede decirse que el territorio expresa el ensamble de ambas estructuras. Ahora bien, si nos proponemos identificar más claramente el lugar que ocupa la noción de territorio en las conceptualizaciones y líneas de investigación de nuestro equipo, podemos afirmar que la misma se encuentra implicada en tres ejes.

El primero de ellos es el que consiste en el estudio de las estrategias de reproducción de los hogares del Conurbano Bonaerense. Dentro del vasto universo de los sectores populares, estudiamos a aquellos que han accedido a la tierra y a la vivienda por fuera de los mecanismos del mercado inmobiliario formal, es decir, aquellos que desarrollan una particular estrategia de acceso al suelo, a la vivienda y a los servicios urbanos. Es precisamente en el territorio en donde se despliegan y adquieren existencia y racionalidad las estrategias de reproducción. En trabajos previos del equipo las hemos deslindado, en principio, en cuatro tipos: estrategias de obtención de bienes de uso, estrategias de obtención de ingresos, estrategias de financiamiento y estrategias de acumulación del fondo de reproducción. "En este sentido, sostenemos que el acceso a financiamiento implica el desarrollo de estrategias que tienen una racionalidad propia y que, por lo tanto, no pueden ser equiparadas con las estrategias de generación de ingresos ni con las de obtención de valores de uso" (Cabrera y Vio, 2014). Asimismo, la estrategia que refiere a la ampliación del fondo de reproducción resulta del entramando de las anteriores y condensa las prácticas dirigidas al sostenimiento y ampliación del capital social o sistema de relaciones en que se inserta el hogar, en las que se pone 
en juego la construcción de vínculos (fuertemente jerarquizados) que ofrecen garantías a la reproducción y retroalimenten las posibilidades de reproducción ampliada de las condiciones de vida (Cabrera y Vio, 2014).

Lo anterior supone la participación consciente de los hogares en sistemas de relaciones en los que se articulan vínculos familiares y vecinales, jerárquicos, y contenidos en lo que llamamos "matriz político-territorial".

Este último es el segundo eje en donde inscribimos la noción de territorio. Las matrices político-territoriales (cada barrio en el que se inscriben los hogares en estudio presenta la propia) son dispositivos de mediación entre los destinatarios y los otorgadores de los recursos provenientes de las políticas sociales. En los territorios estas matrices se convierten en las reales "asignadoras" de las políticas sociales en general. En otras palabras, están fundadas en el amalgamamiento del poder estatal con el de las organizaciones de base y con los poderes individuales de "referentes comunitarios/ barriales". Esta "fusión" de poderes diversos que observamos en ellas es posible a partir de las transferencias de diversos tipos de capital -bienes de uso/capital económico/capital social/ capital simbólico- que son asignados en función de la forma que asume la articulación de la matriz políticoterritorial con los fondos de reproducción de los hogares (Cabrera y Vio, 2014).

Finalmente, el tercer eje en el que el concepto de territorio cobra sentido dentro de las líneas de investigación del PEC, es el referido al estudio de los enclaves productivos y de oficios. En los diferentes trabajos de campo que venimos llevando adelante desde el año 2011, hemos podido observar la conformación de lo que nominamos, a modo de hipótesis, como "enclaves productivos" y "enclaves de oficios” en barrios del Conurbano. Para ser más explícitos, en los barrios de Costa Esperanza e Independencia del partido de San Martín (ambos ubicados en el primer cordón del Conurbano Bonaerense) se ha advertido un sector de la economía popular que, previo a la etapa de la posconvertibilidad, vinculaba su reproducción a actividades de recupero de basura. Ambos barrios se sitúan en las inmediaciones 
del CEAMSE. La tensión entre el uso residencial y sanitario de las fracciones de tierra -propiedad de la Coordinación Ecológica- en las que se emplazan estos barrios, aportó a la conformación de un área de especialización vinculada a la gestión de la basura y al negocio del recupero (Vio, 2018). Es así como durante la posconvertibilidad, en el marco de la matriz que articula sus modos de reproducción, esta actividad cobró mayor centralidad.

Por su parte, en el barrio 22 de enero, ubicado en el partido de La Matanza en el segundo cordón del Conurbano, hemos observado la presencia de pequeños talleres -mayormente dedicados a la actividad textil- que involucran a los trabajadores de la economía popular como eslabones de una cadena productiva más amplia. El barrio, contando con servicios urbanos informales y trabajo no registrado, ofrece las condiciones territoriales necesarias para llevar adelante la actividad y contribuir de este modo a la acumulación de capital de empresas registradas que se proveen de los productos allí elaborados para su posterior comercialización.

Por último, en la investigación realizada por el PEC en 2011 en el barrio San Cayetano-San Blas del partido de San Miguel (segundo cordón del Conurbano), cuya mayoría de habitantes son nacidos en Paraguay (57,6\%), relevamos que priman dos tipos de ocupación. Entre los trabajos no calificados predomina el servicio doméstico (76,4\%), y entre los trabajos calificados, los vinculados a la construcción (63,6\%: albañiles y mamposteros, carpinteros de armar y de blanco, pintores y empapeladores, constructores con técnicas y materiales tradicionales, fontaneros e instaladores de tuberías, electricistas de obras y afines y herreros y forjadores). Podemos decir entonces, que -en los términos de nuestra conceptualización- se trata de una especialización territorial y enclave de oficios, en donde la transmisión del saber ligado a la construcción se encuentra estrechamente vinculada al territorio, a los vínculos, relaciones y posibilidades que éste ofrece.

Es así como, en los tres ejemplos ofrecidos, es posible observar cómo el territorio se halla intrínsecamente ligado a las estrategias de reproducción de los hogares en estudio. Conceptos como economía 
popular, estrategias de reproducción, matrices político-territoriales y territorio, conforman a nuestro entender un esquema conceptual imbricado e inescindible.

Finalmente -y sin ánimo de dar por concluidos o agotados los diálogos entre disciplinas- si hiciésemos el intento de "traducir" algunos aportes teóricos de las ciencias sociales a la práctica proyectual de arquitectos y urbanistas, diríamos en primer lugar que el territorio es un concepto que supone la articulación entre estructura espacial y estructura social.

Sucesivamente, que el estudio de un determinado territorio que toma como criterio de recorte del mismo a la morfología urbana o a sus aspectos físicos, no puede dar cuenta de las complejidades sociales que dicho territorio presenta en un espacio-tiempo ni expresa sus heterogeneidades en términos de condiciones de vida, estrategias de reproducción o matrices político-territoriales. Un recorte geográfico no homogeniza ninguno de los aspectos antes mencionados.

A su vez, el territorio expresa intereses en conflicto por parte de distintos sectores sociales y son los habitantes, entre otros factores intervinientes, quienes "hacen" y moldean cotidianamente y a lo largo del tiempo los territorios que habitan. Por su parte, la escala espacial y los recursos naturales propios de un geoterritorio no son determinantes exclusivos de los límites de un territorio.

Asimismo, el modo de vida de una determinada población expresa espacio, tiempo y necesidades, y éstas últimas son dinámicas, se modifican en el tiempo.

Finalmente, diremos que los mapas y representaciones espaciales son abstracciones en un medio, son construcciones dentro de un tiempo y condiciones dadas, y la existencia humana se inscribe necesariamente en un territorio. De esta forma, el territorio es espacio vivido y constituye una matriz de existencia social, de reproducción; una mediación entre experiencias vividas, relaciones sociales y representaciones construidas por individuos.

Los investigadores en ciencias sociales, así como aquellos dedicados a la planificación urbana tienen ante todo el desafío de poder objetivar su propia posición de clase en la estructura social al momento 
de pensar, investigar y proyectar territorios ocupados por sectores que se ubican en posiciones disímiles a las propias. Tienen el desafío de explicitarse qué concepción o concepciones de territorio están implícitas en su práctica y de hacer de ella un ejercicio de reflexividad constante.

\section{Referencias bibliográficas}

Cabrera, M. C., \& Vio, M. (Eds.). (2014). La trama social de la economía popular, Buenos Aires, Argentina: Espacio Editorial.

Cabrera M. C. \& Vio M. (2014) Enclaves de la economía popular. Territorialidad y estrategias de obtención de ingresos. Ponencia presentada en el taller "Distribución de la riqueza: nuevos escenarios, nuevos paradigmas y alternativas. Una mirada regional e integral, Cátedra Abierta Plan Fénix.

D’Angelo, A. S. (2017). Las estrategias de financiamiento de la economía popular para la producción del hábitat en la posconvertibilidad. Una indagación en Campo Unamuno en el partido de Lomas de Zamora del Conurbano Bonaerense (tesis de maestría). Universidad Nacional de Buenos Aires. Buenos Aires, Argentina

Dematteis, G., \& Governa, F. (2005). Territorio y territorialidad en el desarrollo local. La contribución del modelo SLOT. Boletín de la Asociación de Geógrafos Españoles, (39), 31-58. Recuperado de https://dialnet.unirioja.es/servlet/articulo?codigo=1161234

Faure, A. y Douillet, AC (2005). La acción pública y la cuestión territorial, Francia: PUG

Gutiérrez, A. (2003) "La construcción social de la pobreza. Un análisis desde las categorías de Pierre Bordieu”. Anduli, revista andaluza de ciencias sociales. (2), 29-44.

Jolly, J. F. (2012) "La interdeterminación entre territorio, territorialidad y territorialización de las políticas públicas: hacia una nueva propuesta de esquema para el análisis de las políticas públicas en el territorio”. XVII Congreso Internacional del CLAD sobre la Reforma del Estado y de la Administración Pública, Cartagena, Colombia Recuperado de https://cgcid.org/wp-content/uploads/2015/10/ Territorializaci\%C3\%B3n-de-la-Acci\%C3\%B3n-P\%C3\%BAblica_Jolly.pdf

Monnet J. (2010). "El territorio reticular”. Revista Anthopos, (227), 91-104

Nievas, F. (1994) "Hacia una aproximación crítica a la noción de territorio". Nuevo Espacio. Revista de Sociología, (1), 1-19.

Tomadoni, C. (2008). "A propósito de las nociones de espacio y territorio”. Gestión y Ambiente, 10, (1), 53-66. 
Vio, M. (2017) "Clases populares, economía popular y Conurbano posconvertibilidad. Entre la teoría y la economía popular realmente existente". Ponencia presentada en $X$ Jornadas de Economía Crítica. Buenos Aires, Argentina

Vio, M. (2018) Nada es pesado para llevar a casa: economía popular en la posconvertibilidad. Análisis de las condiciones de vida y estrategias de reproducción social de los hogares que viven de la basura en el partido de San Martín (tesis de doctorado). FLACSO, Buenos Aires, Argentina.

Recepción: 20/11/2018

Aceptación: 12/09/2019 